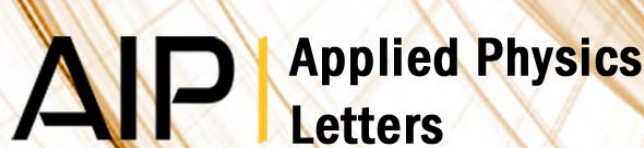

\section{Charging and discharging of graphene in ambient conditions studied with scanning probe microscopy}

A. Verdaguer, M. Cardellach, J. J. Segura, G. M. Sacha, J. Moser et al.

Citation: Appl. Phys. Lett. 94, 233105 (2009); doi: 10.1063/1.3149770

View online: http://dx.doi.org/10.1063/1.3149770

View Table of Contents: http://apl.aip.org/resource/1/APPLAB/v94/i23

Published by the American Institute of Physics.

\section{Related Articles}

Adsorption of small molecules on silver clusters

J. Chem. Phys. 136, 024314 (2012)

Hybrid density functional/molecular mechanics studies on activated adsorption of oxygen on zeolite supported gold monomer

J. Chem. Phys. 135, 244703 (2011)

Modeling intermolecular interactions of physisorbed organic molecules using pair potential calculations

J. Chem. Phys. 135, 234703 (2011)

Adsorption and dissociation of NO on $\operatorname{Ir}(100)$ : A first-principles study

J. Chem. Phys. 135, 204707 (2011)

Combined optical and acoustical method for determination of thickness and porosity of transparent organic layers below the ultra-thin film limit

Rev. Sci. Instrum. 82, 103111 (2011)

\section{Additional information on Appl. Phys. Lett.}

Journal Homepage: http://apl.aip.org/

Journal Information: http://apl.aip.org/about/about_the_journal

Top downloads: http://apl.aip.org/features/most_downloaded

Information for Authors: http://apl.aip.org/authors

\section{ADVERTISEMENT}

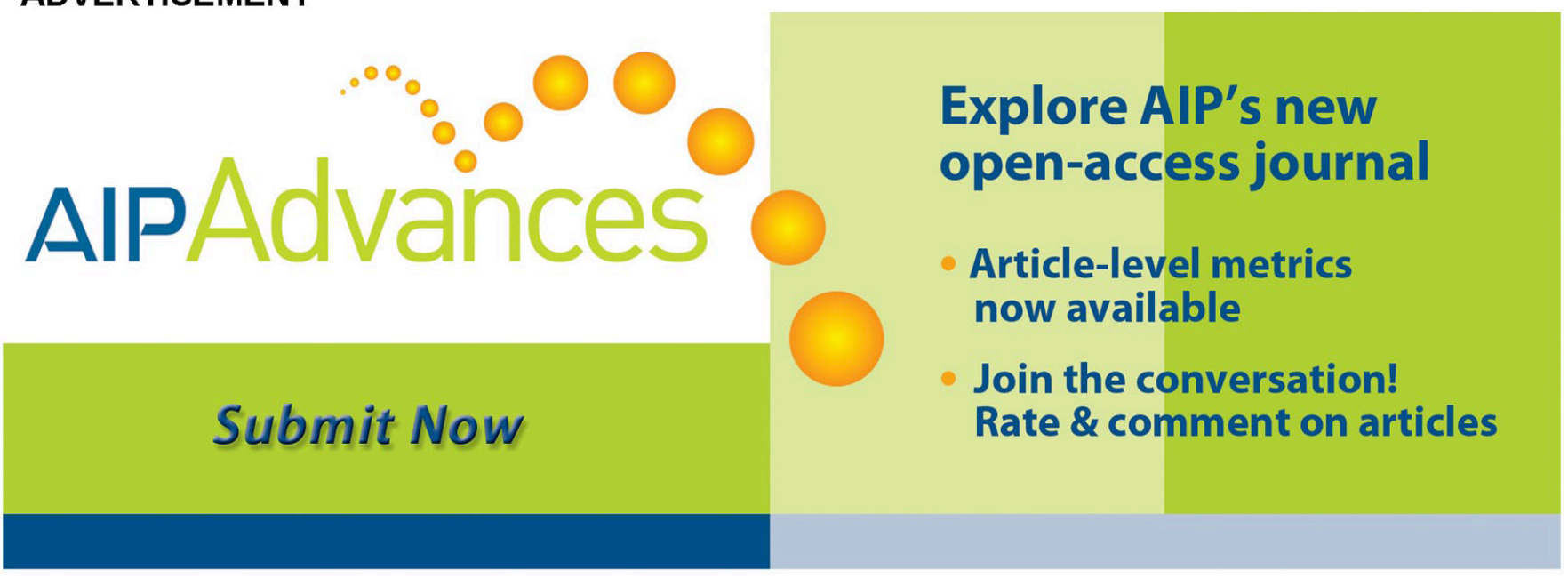




\title{
Charging and discharging of graphene in ambient conditions studied with scanning probe microscopy
}

\author{
A. Verdaguer, ${ }^{1, a)}$ M. Cardellach, ${ }^{1}$ J. J. Segura, ${ }^{1}$ G. M. Sacha, ${ }^{2}$ J. Moser, ${ }^{1}$ M. Zdrojek, ${ }^{1}$ \\ A. Bachtold, ${ }^{1}$ and J. Fraxedas ${ }^{1}$ \\ ${ }^{1}$ Centre d'Investigació en Nanociència i Nanotecnologia (CIN2) (CSIC-ICN), Esfera UAB, \\ Campus de la UAB, Edifici CM-7, 08193-Bellaterra, Catalunya, Spain \\ ${ }^{2}$ Grupo de Neurocomputación Biológica, Escuela Politécnica Superior, Universidad Autónoma de Madrid, \\ Cantoblanco, Madrid E-28049, Spain
}

(Received 24 April 2009; accepted 13 May 2009; published online 9 June 2009)

\begin{abstract}
By means of scanning probe microscopy we are able to inject charges in isolated graphene sheets deposited on $\mathrm{SiO}_{2} / \mathrm{Si}$ wafers and characterize the discharge induced by water in controlled ambient conditions. Contact potential differences between the graphene surface and the probe tip, measured by Kelvin probe microscopy, show a linear relationship with the tip bias during charge injection. The discharge depends on relative humidity and decays exponentially with time constants of the order of tens of minutes. We propose that graphene discharges through the water film adsorbed on the $\mathrm{SiO}_{2}$ surface. (C) 2009 American Institute of Physics. [DOI: 10.1063/1.3149770]
\end{abstract}

Charge injection combined with scanning probe microscopy (SPM) technique provides a powerful tool to study the electrostatic properties of objects in the nanoscale. It allows to measure electrostatic forces in the vicinity of the charged object to gain insight into the distribution of charges within the object, as demonstrated, e.g., in carbon nanotubes ${ }^{1}$ and silicon nanoparticles. ${ }^{2}$ Of equal interest is the use of these techniques to learn about the electrostatic interaction between the object under study and its environment. This is of fundamental importance in surface science. The electronic properties of nanosized objects are strongly affected by the substrate they are deposited on and by molecular adsorbates, which are both difficult to control and require careful characterization. At present, an example attracting huge interest is graphene on silicon oxide. Graphene, a two-dimensional crystal of carbon atoms arranged in a triangular lattice, ${ }^{3}$ is entirely exposed to its environment, which critically limits its performances as an electronic material. ${ }^{4}$ In particular, water and other molecules adsorb on the surface of graphene and can modify its carrier concentration. This property has been used to detect individual gas molecules adsorbed on graphene. ${ }^{5}$

In this letter we present an experimental procedure to charge individual few layer graphene (FLG) films deposited on a $\mathrm{SiO}_{2} / \mathrm{Si}$ wafer using SPM techniques. Once charged the FLG films are characterized using scanning polarization force microscopy (SPFM) and Kelvin probe microscopy (KPM). FLG films are found to discharge into the Si substrate through the water film adsorbed on the $\mathrm{SiO}_{2}$ surface.

All the experiments shown here were carried out at room temperature with a commercial atomic force microscope (Agilent 5500) modified to operate in the SPFM mode. The SPFM operational mode has been reported in detail elsewhere ${ }^{6,7}$ and only a brief description is given here. SPFM is an electrostatic SPM mode in which a conductive tip is set at about $10-20 \mathrm{~nm}$ from the surface and biased to a few volts. ${ }^{8}$ Together with KPM, it allows one to obtain simulta-

\footnotetext{
a) Author to whom correspondence should be addressed. Electronic mail: albert.verdaguer.icn@uab.cat.
}

neously information about topography, polarizability, and contact potential difference (CPD) between the tip and the surface.

The microscope head was enclosed in a glove box to control the relative humidity $(\mathrm{RH})$ of the environment. FLG films on silicon wafers were prepared using the conventional micromechanical exfoliation technique, ${ }^{9}$ where a flake of bulk Kish graphite is repeatedly cleaved with an adhesive tape and pressed down onto a silicon wafer coated with 280 $\mathrm{nm}$ thermally grown silicon oxide.

Charge injection is performed as follows. The silicon substrate is connected to ground during the experiment. An image in contact mode of the FLG film is taken with the tip grounded [Fig. 1(a), top left]. Then, the tip is placed above the center of the FLG film, brought into contact with it and a bias $\left(V_{\text {inj }}\right)$ is applied to the tip within the -10 to $10 \mathrm{~V}$ range [Fig. 1(a), top right]. After a few minutes the tip is retracted to about $200 \mathrm{~nm}$ from the surface, keeping the tip biased [Fig. 1(a), lower left]. With this procedure the FLG film becomes charged acquiring an initial electric potential $V_{0}$, different from $V_{\text {inj, }}$, which evolves with time as discussed below. Once the tip is retracted from the surface, $V_{\text {inj }}$ on the tip is switched off and a dual SPFM+KPM image is taken [Fig. 1(a), lower right]. Using this method we can guarantee noncontact conditions during all throughout the experiment on the FLG film and avoid the formation of a water neck between the tip and the sample ${ }^{10}$ that would perturb the discharge process. An image in contact mode with the tip grounded can be performed to discharge the FLG, as can be seen by comparing KPM images before charging and after discharging.

Figure 1(b) shows SPFM (top left) and KPM (top right) images of a few microns wide FLG sample. In the SPFM images the FLG films show a high contrast (apparent height) due to the huge difference in the dielectric constant $(\varepsilon)$ between $\mathrm{SiO}_{2}$ and the FLG films. ${ }^{11}$ The KPM image, taken simultaneously with the SPFM image, shows a CPD slightly less positive in the FLG region compared to the $\mathrm{SiO}_{2}$ substrate, in agreement with recent results. ${ }^{12}$ In Fig. 1(b) we show KPM images of the FLG film after charging it at RH 
(a)
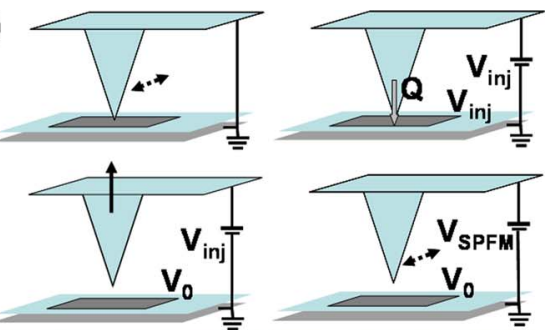

(b)

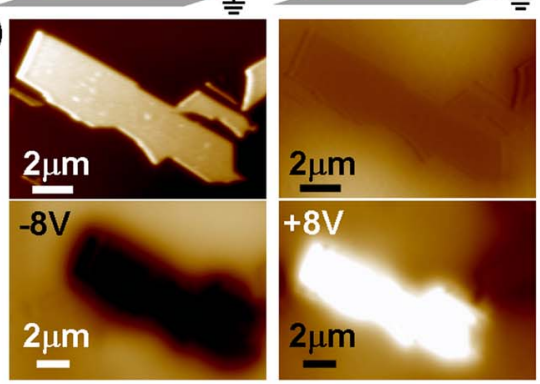

(c)

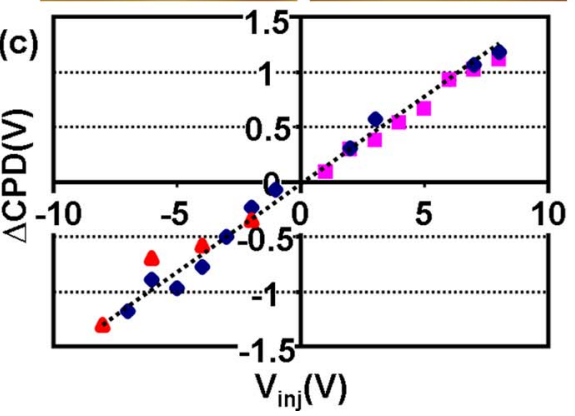

FIG. 1. (Color online) (a) Scheme of the experimental charging and measurement procedure used. (b) SPFM (top left) and KPM (top right) images of a FLG film before charging at low RH. Bottom: KPM images of the same film after charging to (left) $-8 \mathrm{~V}$ and (right) $+8 \mathrm{~V}$. (c) $\triangle \mathrm{CPD}$ CPD after charge injection-CPD discharged) vs voltage applied to the tip during the charging process $\left(V_{\text {inj }}\right)$ measured from KPM taken after charge injection. $\Delta$ CPD appears to be proportional to $V_{\text {inj }}$. Different symbols denote different experiments with different tips and different samples. All experiments were performed at $\mathrm{RH}<5 \%$.

$<5 \%$ with $-8 \mathrm{~V}$ (down left) and $+8 \mathrm{~V}$ (lower right), respectively. In the KPM images the FLG film charged with $+8 \mathrm{~V}(-8 \mathrm{~V})$ show a bright (dark) CPD contrast.

In the following, we investigate the charge and discharge of FLG films by measuring the CPD between the FLG film and the tip. We first characterize our experimental setup by measuring the dependence of CPD on $V_{\text {inj. }}$. We then study the influence of humidity in the environment, which brings us to consider various discharge mechanisms. In Fig. 1(c) we plot the difference between CPD $(\triangle \mathrm{CPD})$ measured on three different FLG films before and after charging as a function of $V_{\text {inj }}$ at $\mathrm{RH}<5 \%$, showing a linear behavior with a slope $s \sim 0.1\left(\Delta \mathrm{CPD} \approx s \cdot V_{\text {inj }}\right)$. Note that after each charge injection and measurement of $\triangle \mathrm{CPD}$ graphene is brought back to its initial state (by discharging) in order to avoid any charge accumulation effect. Different contributions can influence the charging process and the subsequent $\triangle \mathrm{CPD}$ measurements: (i) a poor tip-FLG contact, (ii) the geometry of the tip-FLG system, (iii) the discharging of the FLG film into the environment and (iv) the loss of some of the injected charge during the retraction of the tip. In order to check the tip-FLG contact, the contact time between the tip and the FLG film has been increased up to $30 \mathrm{~min}$ (the usual contact time was $\sim 1-3 \mathrm{~min}$ ). Additionally, the increase in the load applied to the tip during the charging process has been verified. Both parameters had no effect on the measured $\triangle \mathrm{CPD}$. This indi-

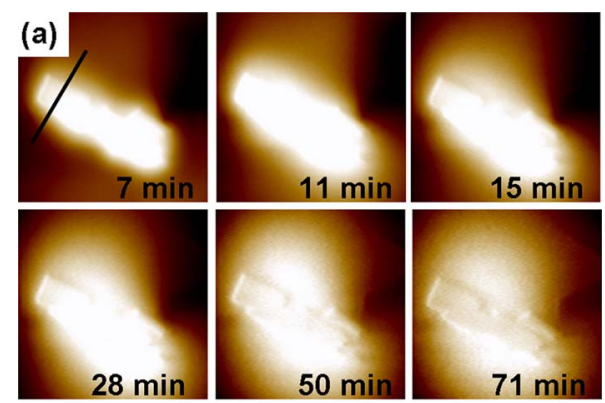

(b)

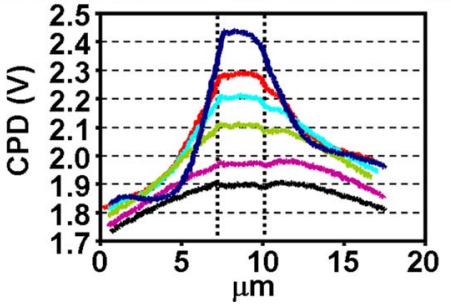

FIG. 2. (Color online) (a) Time evolution of KPM images of a FLG film charged at $+8 \mathrm{~V}$ and at $30 \% \mathrm{RH}$. (b) Cross section profiles of the same images.

cates that the electrical contact between the FLG film and the tip was good enough. In addition, the high reproducibility of the obtained slope over several independent experiments rules out an uncontrolled loss of charge during injection.

The slope $s$ strongly depends on the geometry of the system represented by the tip and the FLG film. We measured $s \sim 0.1$ for FLG films with areas ranging from 6 to $30 \mu \mathrm{m}^{2}$. Measurements performed on much larger FLG films (in the order of the tip dimensions, 200-400 $\mu \mathrm{m}^{2}$ ) still show a linear behavior but with $s \sim 0.3$. Thus, to control quantitatively the charging process, one should take into account the influence of the full cantilever-tip-graphene geometry.

CPD on a charged FLG film is not constant with time and FLG films discharge with a decay rate that strongly depends on the RH of the environment. In Fig. 2(a) we show the time evolution of KPM images of a FLG film charged at $8 \mathrm{~V}$ and at $30 \% \mathrm{RH}$. The images show how the positive charge spreads over the silicon substrate while the initially confined FLG film discharges. The evolution of a cross section is shown in Fig. 2(b). The CPD on the FLG film decreases and CPD values on the $\mathrm{SiO}_{2}$ surrounding the film increase due to positive charges that are moving away from the FLG film and toward the substrate. It is well known that a clean $\mathrm{SiO}_{2}$ surface rich in $\mathrm{OH}$ superficial groups is a very hydrophilic surface and a few monolayers of water molecules are expected to be adsorbed on the surface for a wide RH range. ${ }^{13}$ Thus, the main discharge process would be through water molecules adsorbed on the FLG film itself ${ }^{14}$ and the water film on the $\mathrm{SiO}_{2}$ substrate. At $t=28 \mathrm{~min}$ in Fig. 2 the CPD on the FLG and the substrate area films surrounding it level at the same value. From this time on the entire CPD profile lowers with time with minimal changes in its shape. This behavior clearly suggests that the slower discharge of the water film surrounding the FLG film is limiting the faster migration of charges from the FLG film to the water film.

The time evolution of the mean CPD measured on the FLG film at different RH conditions exhibits an exponentiallike decay [Fig. 3(a)]. Only the data taken at 50\% RH decays to the CPD value of the FLG film before charge injection 


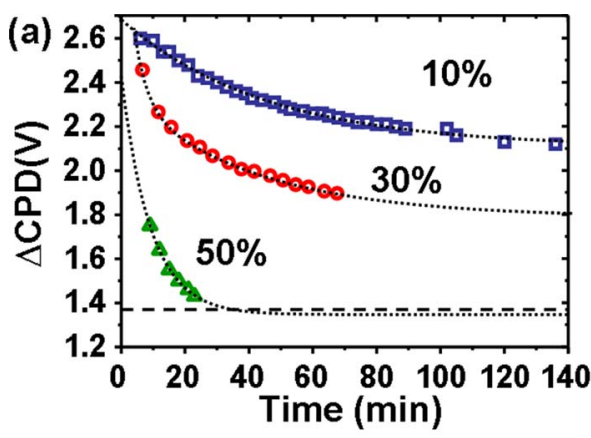

(b)

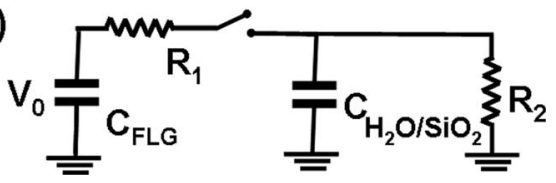

FIG. 3. (Color online) (a) Equivalent $R C$ circuit modeling the discharge of the FLG film. Some of the components of the circuit can be neglected at low humidity $\left(R_{2}\right)$ and at high humidity $\left(C_{\mathrm{H}_{2} \mathrm{O} / \mathrm{SiO}_{2}}\right)$. (b) Evolution with time of the FLG film CPD value after charging it at $+8 \mathrm{~V}$ for $10 \%, 30 \%$, and $50 \%$ RH. Exponential decays used to fit the data [Eqs. (1) and (3)] are shown and the CPD value of the discharged FLG film is marked $(\sim 1.4 \mathrm{~V})$.

$(\sim 1.4 \mathrm{~V}$ for that particular sample) for the times measured. Very similar decays were obtained for both positive and negative voltages.

In order to explain our results we model our system as an equivalent circuit formed by capacitors and resistances [Fig. 3(b)]. One capacitor $\left(C_{\mathrm{FLG}}\right)$ is formed by the FLG film

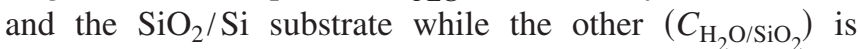
formed by the water film adsorbed on the $\mathrm{SiO}_{2}$ and the $\mathrm{SiO}_{2} / \mathrm{Si}$ substrates (the $\mathrm{Si}$ substrate is grounded). $R_{1}$ represents the resistance that limits the flow of charges on the FLG film to the water film. $R_{2}$ models the discharge of the water film mainly through the diffusion of the water molecules on the $\mathrm{SiO}_{2}$ surface. The solution of the differential equations of the circuit is in the form

$$
V_{\mathrm{FLG}}(t)=A_{1} e^{m_{1} t}+A_{2} e^{m_{2} t},
$$

where

$$
\begin{aligned}
m_{1,2}= & -\frac{1}{2}\left[\frac{1}{C_{1} R_{1}}+\frac{1}{C_{2} R_{1}}\right. \\
& \left.+\frac{1}{C_{2} R_{2}}\right] \pm \frac{1}{2} \sqrt{\left[\frac{1}{C_{1} R_{1}}+\frac{1}{C_{2} R_{1}}+\frac{1}{C_{2} R_{2}}\right]^{2}-4 \frac{1}{C_{1} C_{2} R_{1} R_{2}}},
\end{aligned}
$$

and $V_{\mathrm{FLG}}(0)=A_{1}+A_{2} \equiv V_{0}$.

At $10 \%$ RH we can simplify the model by considering that $R_{2} \rightarrow \infty$ and can thus be ignored. We then obtain that

$$
V_{\mathrm{FLG}}(t)=\frac{C_{T} V_{0}}{C_{\mathrm{H}_{2} \mathrm{O} / \mathrm{SiO}_{2}}}+\frac{C_{T} V_{0}}{C_{\mathrm{FLG}}} e^{-t / R_{1} C_{T}}
$$

where $C_{T}$ stands for the total capacitance $\left(1 / C_{T}=1 / C_{\mathrm{FLG}}\right.$ $\left.+1 / C_{\mathrm{H}_{2} \mathrm{O} / \mathrm{SiO}_{2}}\right)$ and $V_{0}$ is the initial voltage at the $C_{\mathrm{FLG}}$ capacitor. Equation (1) shows the voltage decay for the FLG film going from $V_{0}$ to $V_{F}=C_{T} V_{0} / C_{\mathrm{H}_{2} \mathrm{O} / \mathrm{SiO}_{2}}$ with a time constant $R_{1} C_{T}$. Both $R_{1}$ and $C_{T}$ depend on RH. The increase in water molecules on the $\mathrm{SiO}_{2}$ surface in contact with the FLG film will increase the ratio of charge transfer between the FLG and the water film, thereby lowering the resistance in our model. The amount of water adsorbed on $\mathrm{SiO}_{2}$ would clearly affect $C_{\mathrm{H}_{2} \mathrm{O} / \mathrm{SiO}_{2}}$ and water adsorbed on or below the
FLG film could also modify $C_{\text {FLG }}$. The experimental data fitted with this model is shown in Fig. 3(a) with a time constant of $\sim 50 \mathrm{~min}$. At high humidity there is a high diffusion of charges on the surface and we can consider that $R_{2}$ is so small that no charge is accumulated in $C_{\mathrm{H}_{2} \mathrm{O} / \mathrm{SiO}_{2}}$. The equivalent $R C$ circuit is thus simplified by a circuit composed of a capacitor $C_{\mathrm{FLG}}$ that discharges through a resistance $R_{1}$. The CPD value decays exponentially to its precharging value [1.4 $\mathrm{V}$ for $\mathrm{RH}=50 \%$ with a time constant of $\sim 10 \mathrm{~min}$, in Fig. 3(a)]. At intermediate humidity the discharge of the FLG film depends strongly on the charge accumulated in the water surrounding it and how fast these charges diffuse away from the FLG film. In our model that means that none of the processes are dominating the discharging and both $R_{1}$ and $R_{2}$ must be taken in account. Data taken at 30\% RH [Fig. 3(a)] can only be fitted then using the two exponential decays shown in Eqs. (1) and (2).

In conclusion, we have presented an experimental procedure to charge individual FLG films deposited on a $\mathrm{SiO}_{2} / \mathrm{Si}$ wafer using SPM. We have found that CPD measured on the FLG film using KPM shows a linear relationship with the voltage applied to the tip during the charge injection for both negative and positive voltages ranging from -10 to $+10 \mathrm{~V}$. Discharge of FLG films has been studied at different RH conditions. We have found that CPD follows RH dependent exponential decays with time. We have finally shown that FLG films discharge mainly through the water layer adsorbed on the $\mathrm{SiO}_{2}$ surface.

This work was supported by the Ministerio de Educación y Ciencia (MEC), Spain, through Grant No. FIS2006-12117C04-01, and by the EXPLORA program NAN2007-29375-E. We thank J. L. Masa-Campos and J. J. Sáenz for their interesting discussions on the electrostatics of our system.

${ }^{1}$ M. Zdrojek, T. Mélin, H. Diesinger, D. Stiévenard, W. Gebicki, and L. Adamowicz, J. Appl. Phys. 100, 114326 (2006).

${ }^{2}$ T. Mélin, D. Deresmes, and D. Stiévenard, Appl. Phys. Lett. 81, 5054 (2002).

${ }^{3}$ A. K. Geim and K. S. Novoselov, Nature Mater. 6, 183 (2007).

${ }^{4}$ J.-H. Chen, C. Jang, S. Adam, M. S. Fuhrer, E. D. Williams, and M. Ishigami, Nat. Phys. 4, 377 (2008)

${ }^{5}$ F. Schedin, A. K. Geim, S. V. Morozov, E. W. Hill, P. Blake, M. I. Katnelson, and K. S. Novoselov, Nature Mater. 6, 652 (2007).

${ }^{6} \mathrm{~L}$. Xu and M. Salmeron, in Nano-Surface Chemistry, edited by M. Rosoff (Dekker, New York, 2001), Chap. 6.

${ }^{7}$ A. Verdaguer, G. M. Sacha, H. Bluhm, and M. Salmeron, Chem. Rev. (Washington, D.C.) 106, 1478 (2006).

${ }^{8}$ The external voltage applied to the tip $V_{\text {tip SPFM }}$, has both a dc and an ac component, $V_{\mathrm{dc}}+V_{\mathrm{ac}} \sin (\omega t)$. The tip oscillates in response to the electrostatic forces created by $V_{\mathrm{ac}} . V_{\mathrm{dc}}$ is used to null the first harmonic component of the tip oscillation, thus providing a direct measurement of the tip-surface contact potential difference as in KPM.

${ }^{9}$ K. S. Novoselov, A. K. Geim, S. V. Morozov, D. Jiang, Y. Zhang, S. V. Dubonos, I. V. Grigorieva, and A. A. Firsov, Science 306, 666 (2004).

${ }^{10}$ G. M. Sacha, A. Verdaguer, and M. Salmeron, J. Phys. Chem. B 110, 14870 (2006).

${ }^{11}$ G. M. Sacha, M. Cardellach, J. J. Segura, J. Moser, A. Bachtold, J. Fraxedas, and A. Verdaguer, "Influence of the macroscopic shape of the tip on the contrast in scanning polarization force microscopy," Nanotechnology (to be published).

${ }^{12}$ S. S. Datta, D. R. Strachan, E. J. Mele, and A. T. Charlie Johnson, Nano Lett. 9, 7 (2009).

${ }^{13}$ A. Verdaguer, C. Weis, G. Oncins, G. Ketteler, H. Bluhm, and M. Salmeron, Langmuir 23, 9699 (2007).

${ }^{14}$ J. Moser, A. Verdaguer, D. Jiménez, A. Barreiro, and A. Bachtold, Appl. Phys. Lett. 92, 123507 (2008). 